\title{
Correlation Between the 6-Min Walk Test and Exercise Tolerance Test in Cardiac Rehabilitation After Coronary Artery Bypass Grafting: A Cross-sectional Study
}

\author{
Maryam A. Saba (D) - Shahin Goharpey (D) - Behrouz Attarbashi Moghadam • \\ Reza Salehi · Mostafa Nejatian
}

Received: November 13, 2020 / Accepted: January 6, 2021 / Published online: February 13, 2021

(C) The Author(s) 2021

\begin{abstract}
Introduction: Exercise capacity and quality of life are the main outcomes in cardiac rehabilitation (CR). Exercise capacity is one of the important prognostic and diagnostic measures acquired by the exercise tolerance test (ETT). The 6-min walk test (6MWT), as a functional walking test, is another tool for assessing exercise capacity.

Methods: Eighty postoperative coronary artery bypass grafting (CABG) participants admitted to three CR units were recruited for this cross-sectional and multicenter study, based on convenient non-probability sampling. All participants
\end{abstract}

M. A. Saba · S. Goharpey ( $\square)$

Musculoskeletal Rehabilitation Research Center, Ahvaz Jundishapur University of Medical Sciences, Ahvaz, Iran

e-mail: shgoharpey@yahoo.com

B. Attarbashi Moghadam

Physical Therapy Department, School of

Rehabilitation, Tehran University of Medical

Sciences, Tehran, Iran

R. Salehi

Rehabilitation Research Center, Department of

Rehabilitation Management, School of

Rehabilitation Sciences, Iran University of Medical

Sciences, Tehran, Iran

M. Nejatian

Cardiac Rehabilitation Department of Tehran Heart

Center, Tehran University of Medical Science,

Tehran, Iran performed an ETT and two repeated 6MWTs. Maximum heart rate (HR), maximum blood pressure (BP), maximal oxygen uptake $\left(\mathrm{VO} 2_{\text {max }}\right)$, and peak metabolic equivalents of tasks (MET) during the tests and also the 6-min walk distance (6MWD) were the outcome measures.

Results: The mean age of all participants was 62.13 (7.12) years and 80\% were male. Pearson correlation showed that maximum HR $(r(78)=0.67, P<0.001)$ and maximum systolic BP $(r(78)=0.57, P<0.001)$ during the 6MWT correlated moderately with those achieved during ETT. Maximum HR in the 6MWT corresponded to $86 \%$ of that achieved during ETT. The 6MWD showed a strong positive correlation with peak MET estimated during ETT as a measure of exercise capacity $(r=0.77$, $P<0.001)$.

Conclusions: The results from this study suggest that the 6MWT is a valid tool for assessing functional capacity for prescribing exercise in a group of postoperative CABG participants admitted to CR units. This finding is helpful for healthcare professionals and for patients, as the test is easy to administer and also well tolerated by patients.

Trial Registration Number: PHT-9923.

Keywords: Cardiac rehabilitation; Exercise capacity; Metabolic equivalents; The 6-min walk test 


\section{Key Summary Points}

The 6-min walk test (6MWT), as a functional walking test, is a tool for assessing exercise capacity that nowadays with the growing attention to functional and inexpensive outcome measures in therapeutic interventions, and for its ease of performance, has become a more applicable approach.

Assessing the correlation of pertinent variables for the 6MWT and exercise tolerance test (ETT), indicating 6MWT validity in cardiac rehabilitation (CR) (and also investigating the relationship between 6MWT distance and peak metabolic equivalents of tasks (MET) estimated during ETT) were the aim of this study.

The 6MWT distance can be used as a valid CR outcome measure, having several advantages over the treadmill test, which correlates well with a common criterion in CR centers for exercise capacity means peak MET estimated during ETT.

The results from this study suggest that the 6MWT is an effective tool for assessing functional capacity for prescribing exercise and can be used several times during the sessions for decision-making about ongoing treatment processes and program intensity and has the potential to be an alternative (not a substitute for) ETT.

\section{DIGITAL FEATURES}

This article is published with digital features, including a summary slide, to facilitate understanding of the article. To view digital features for this article go to https://doi.org/10.6084/ m9.figshare.13502655.

\section{INTRODUCTION}

Exercise capacity and quality of life are the main outcomes in cardiac rehabilitation (CR) $[1,2]$. For functional exercise capacity evaluation, there are various modalities available; some of them assess all the systems involved in exercise and the pathophysiology mechanisms, while others only provide basic information. The modality of the preference is chosen based on clinical decision-making and available tools and circumstances [3]. The cardiopulmonary exercise test (CPET) is one of these modalities that measures maximal oxygen uptake $\left(\mathrm{VO} 2_{\max }\right)$ directly with a gas analyzer, which seems to be the best index of aerobic capacity and the gold standard for cardiorespiratory fitness [4]. However, this technique is not widely used due to its expense, the need for sophisticated equipment, and not being well tolerated by elderly patients or those with comorbidities $[5,6]$. In some centers after revascularization and myocardial infarction, an exercise tolerance test (ETT) is performed with modified Bruce protocol, a less strenuous protocol in comparison to Bruce protocol [7], without a gas analyzer. Exercise capacity is one of the important prognostic and diagnostic measures acquired by ETT that is an estimate of $\mathrm{VO} 2_{\max }$, measured in units of metabolic equivalents (MET) [8]. One MET is the amount of oxygen consumption required in a resting state, and equals $3.5 \mathrm{ml}$ of oxygen per kilogram of body weight per minute for an average adult $[9,10]$. Peak MET is estimated according to grade and speed attained on a treadmill at the maximum exercise tolerance. Due to variation in protocols and conditions, for calculating the exercise capacity, the amount of work performed in METs is preferred to the number of minutes of exercise [10].

The 6-min walk test (6MWT), as a functional walking test, is another tool for assessing exercise capacity that nowadays with the growing attention to functional and inexpensive outcome measures in therapeutic interventions, and for its ease of performance, has become a more applicable approach. A review article on functional walking tests in the cardiorespiratory domain showed that the 6MWT is easy to administer and well tolerated [11]. Another 
advantage of the 6MWT over the treadmill test is that walking is a natural daily activity and so can reflect the real situation, and even the elderly and patients with musculoskeletal disorders like knee arthrosis can perform it without the fear of instability or falling off the treadmill [12]. The protocol used for the elderly and deconditioned individuals in ETT is usually less energy-consuming $[7,10]$. Moreover, the 6MWT for middle-aged and the elderly is considered a moderate- to high-intensity activity $[6,13]$. Besides, pain, long bed rest, and respiratory limitation after sternotomy, which is common in most revascularization patients referred to CR centers, [14] may exceed the level of exertion for 6MWT. Therefore, the intensity of these tests in CR seems to be somewhat related. The primary outcome in the 6MWT is the 6-min walk distance (6MWD), which can be used for exercise prescription and is also suitable for pre- and post-CR outcome assessment [15]. MET is another variable that can be estimated by the $6 \mathrm{MWT}$ through a calculation that converts 6-min walking speed into a MET level $([3.5+(6 \mathrm{MWT}$ speed $\times 0.1)] / 3.5)[16,17]$.

One of the major group of patients referred to $\mathrm{CR}$ centers are those after coronary artery bypass grafting (CABG) $[14,18]$. In some centers, ETT is performed, but in other smaller centers with more limitations, no entry graded exercise test is done. Therefore, there is a need for functional tests that can be performed before starting the rehabilitation for prescribing the exercises with appropriate intensity as well as assessing the outcomes. Despite the prevalence of referred postoperative CABG patients to the $\mathrm{CR}$ centers, there has been no research on assessing the correlation of pertinent variables for the 6MWT and ETT yet. Investigating the relationship between 6MWD and peak MET estimated during ETT is the other aim of this study. By existence of this associations, 6MWT may be assumed as a cost-effective tool, especially in small rehabilitation centers, for prescribing exercise and assessing the CR outcomes.

\section{METHODS}

Eighty postoperative CABG participants were admitted to three CR units; Imam Khomeini hospital in Ahvaz, Tehran Heart Center, and Shariati Hospital, which both are located in Tehran. The participants were recruited for this cross-sectional and multicenter study based on convenient non-probability sampling. The inclusion criteria were age above 40 years, CABG surgery during the last 2 months, an ETT report (therefore, having no problems interfering with treadmill walking), and no serious psychiatric disorders. Unwillingness to cooperate and contraindications for the 6MWT including unstable angina and myocardial infarction in the previous month, resting heart rate (HR) of more than $120 \mathrm{ppm}$, a systolic blood pressure (BP) of more than $180 \mathrm{mmHg}$, and a diastolic BP of more than $100 \mathrm{mmHg}$ [3], were the exclusion criteria. Before the experiment, the aim and the protocol of the study was explained to the participants, and then an informed consent form was signed. Demographic data [age, sex, body mass index (BMI)] and clinical information (ejection fraction (EF), diabetes, high BP and hypercholesterolemia as risk factors) were recorded. The Medical Ethics Committee of the Ahvaz Jundishapur University of Medical Sciences has approved the study protocols (IR.AJUMS.REC.1399.582). Written informed consent was obtained from all of the study subjects. The study was performed in accordance with the Declaration of Helsinki 1964 and its later amendments.

All participants performed an ETT and two repeated 6MWTs. The 6MWT was conducted according to a standard protocol on a flat, hard, and measured corridor, with cones marking the turning points [3] by a physiotherapist. The distance of the hallway was modified from 30 to $10 \mathrm{~m}$ due to space constraints in the centers, and such distance is already being used for 6MWT [19]. Before the test, the participants' resting HR and BP were monitored and recorded using a portable device (MySign ${ }^{\circledR}$ S, Envitec Co.) and an immovable one (Masimo set, NOVIN S1800, Saadat Co.), respectively. Immediately after completion of the 6MWT, peak exercise 
data including $\mathrm{HR}, \mathrm{BP}$, rating of perceived exertion (RPE) (the participants rating themselves on a Borg RPE scale from 6 to 20), and the total distance walked in meters was measured. Other measured parameters included angina and dyspnea ratings, and pulse oximetry $\left(\mathrm{SpO}_{2}\right)$ recordings that were obtained primarily for participants' monitoring and safety purposes, were not formally collected or analyzed. Participants were instructed to walk at their own pace through the hallway while attempting to cover as much ground as possible in $6 \mathrm{~min}$, and take rest periods if necessary, but resume walking as soon as they could. Participants were encouraged with the standardized statements and the remaining time was called out every 2 min. An electronic timer with a buzzer that sounded the completion of the 6 min was used. Participants who became symptomatic (angina, severe dyspnea) were instructed to stop walking and the test was discontinued. Each subject completed two repeated 6MWTs during the same day, with a seated rest of 30 min between trials until HR, BP, and RPE returned to baseline values. Data from the test with more distance were used for the analysis.

The ETT was conducted in a cardiologist-supervised assessment laboratory about 1-6 days before the 6MWT according to the routine clinical practice setting in the centers, and means patients were usually admitted to cardiac rehabilitation units within a week after performing ETT. Subjects performed ETT with modified Bruce protocol and the starting point (i.e., stage 1) was $1.7 \mathrm{mph}$ at a $0 \%$ grade. The treadmill grade and then speed were increased gradually and subjects were encouraged to exercise until exhaustion. Test termination criteria consisted of the most accepted indications $[4,7]$. HR was monitored with standard electrocardiographic leads, and BP was measured with a standard cuff sphygmomanometer (Masimo set, NOVIN S1800, Saadat Co.) before starting the test and after each stage. The cardiologist provided written documentation for ETT termination and the ETT report should be added to the participants' medical charts. Therefore, the data in the report, including maximum $\mathrm{HR}$, maximum $\mathrm{BP}, \mathrm{VO}_{2} \mathrm{max}$, and peak MET, were used for the final analysis.
Statistical Package for the Social Sciences (SPSS) was used for data analysis and $P<0.05$ was considered as statistically significant. Graphs were plotted using MedCalc statistical software.

\section{RESULTS}

A total of 80 postoperative CABG participants completed the study before starting the rehabilitation. None of the participants rested during the 6MWT and no complications occurred. Data were normally distributed and analyzed using descriptive statistics, Pearson correlation, and linear regression. Demographic and clinical characteristics are presented in Table 1 . The mean age of all participants was $62.13 \pm 7.12$ years, $80.0 \%$ were male, more than $20 \%$ had comorbidities such as diabetes, and approximately half had left ventricular $\mathrm{EF}<50 \%$. Detailed results for $6 \mathrm{MWT}$ and ETT are presented in Table 2. Maximum HR during the 6MWT corresponded to $86.03 \pm 0.08 \%$ of that achieved during ETT. Pearson product moment correlation was run to assess the relationship between pertinent variables achieved during 6MWT and ETT. Strong and significant correlation was found between MET estimated during 6MWT and ETT $(r(78)=0.77, P<0.001)$, as well as between $\mathrm{VO}_{2 \max }$ estimated during $6 \mathrm{MWT}$ and ETT $(r(78)=0.78, P<0.001)$. There was a moderate and significant correlation between maximum HR obtained during 6MWT and ETT $(r(78)=0.67, P<0.001)$. The correlation between maximum systolic BP achieved during 6MWT and ETT was moderate and significant $(r(78)=0.57, P<0.001)$. Using simple linear regression to assess the relation, 6MWD showed a strong positive correlation with peak MET estimated during ETT as a measure of exercise capacity $\left(r=0.77, r^{2}=0.59\right.$, standardized $\beta$ coefficient $=0.77, P<0.0001$; Fig. 1$)$.

\section{DISCUSSION}

Assessing the correlation of pertinent variables for the 6MWT and ETT indicating 6MWT validity in $\mathrm{CR}$ and also investigating the 
Table 1 Demographic and clinical characteristics [mean (standard deviation); or frequency (\%)] of all patients $(n=80)$

Characteristics of participants

Age (years)

Gender

Male

Female

Weight

Height

Body mass index (BMI)

Baseline heart rate (beats/min)

Baseline systolic blood pressure $(\mathrm{mmHg})$

$\mathrm{SpO}_{2}(\%)$

Ejection fraction $(\mathrm{EF})$

Diabetes

Hypertension

Hypercholesterolemia

Obesity (BMI $\geq 30$ )
Mean (standard deviation); or $N$ (frequency)

$62.13(7.12)$

$$
\begin{aligned}
& 64(80.0 \%) \\
& 16(20.0 \%) \\
& 73.83(11.20) \\
& 166.87(7.87) \\
& 26.51(3.69) \\
& 81.54(8.02) \\
& 121.71(10.14) \\
& 95.52(2.73) \\
&<50: 38(47.4 \%) \\
& \geq 50: 42(52.6 \%) \\
& 35(43.8 \%) \\
& 26(32.5 \%) \\
& 21(26.3 \%) \\
& 16(20 \%)
\end{aligned}
$$

Table 2 Mean and standard deviation (SD) of pertinent variables for 6MWT and ETT $(n=80)$

\begin{tabular}{lcc}
\hline & $\begin{array}{l}\text { 6MWT performance characteristics; } \\
\text { mean }(\text { SD) }\end{array}$ & $\begin{array}{l}\text { ETT performance characteristics; } \\
\text { mean (SD) }\end{array}$ \\
\hline Estimated peak MET & $3.23(0.2)$ & $6.44(1.8)$ \\
Estimated $\mathrm{VO}_{\max }$ & $11.30(0.9)$ & $23.40(6.7)$ \\
Maximum heart rate (beats/min) & $105.61(10.9)$ & $123.61(15.4)$ \\
$\begin{array}{l}\text { Maximal systolic blood pressure } \\
(\mathrm{mmHg})\end{array}$ & $133.75(13.4)$ & $141.93(10.8)$ \\
\hline
\end{tabular}

$E T T$ exercise tolerance test, $6 M W T$ 6-min walk test

relationship between 6MWD and peak MET estimated during ETT were the aim of this study. The main result in this study was a strong positive and significant relationship between 6MWD and peak MET estimated during ETT as a measure of exercise capacity. The purpose of measuring functional exercise capacity is to assess the function of body systems including breathing, cardiovascular, neurophysiological, and musculoskeletal all together. Maximum functional capacity achieved during each test, which is commonly considered as an estimation of aerobic capacity, is one of the best predictors of risk in patients with a high risk of 


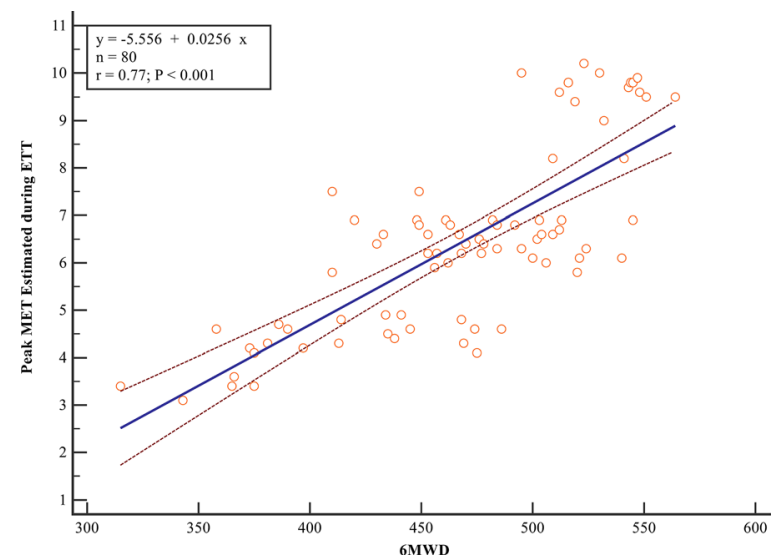

Fig. 1 Scatterplot of 6MWD and peak MET estimated during $\operatorname{ETT}(n=80)$

cardiovascular disease, and a meta-analysis study reported that each increase in MET causes a $13-15 \%$ decrease in mortality and cardiovascular events [10]. To the best of our knowledge, no studies have investigated the relationship between 6MWD and peak MET estimated during ETT yet. However, results demonstrating linear correlations between the 6MWD and $\mathrm{VO} 2_{\max }$ measured on CPET have been described in several previous studies $[6,20,21]$. Sousa et al.'s study showed a linear relationship with correlation coefficient of $r=0.70$ between distance covered at the 6MWT and estimated $\mathrm{VO} 2_{\max }$ reached during the ETT in 24 patients with permanent cardiac pacemakers [22]. The findings of this study are also similar to those in Kristjánsdóttir et al.'s study [12] that showed a strong significant correlation between 6MWD and the maximum power (watt $/ \mathrm{kg}$ ) obtained during the symptom limited graded exercise $(r=0.93)$. Participants were ten elderly patients who underwent cardiac surgery 3 months earlier and also passed a CR program prior to entering the study, and the tool that was used as graded exercise test was a stationary bicycle. The difference between the nature of the test (a stationary bicycle in comparison to treadmill graded test in the present study) may be one reason for the higher $r$ coefficient. The other explanation may be that in their study, the 6MWT was administered in $35-\mathrm{m}$ corridor. A strong significant relationship has been shown in this study, which indicates that the 6MWD can be used as a valid CR outcome measure with all its advantages previously discussed, that is correlated well with a common criterion in some CR centers for exercise capacity means peak MET estimated during ETT.

The other main result of this study showed a significant association between pertinent variables of 6MWT and ETT. The maximum HR $(r=0.67)$ and maximum systolic BP $(r=0.57)$ during 6MWT correlated moderately with those achieved during ETT. The higher mean of these variables during ETT in comparison to $6 \mathrm{MWT}$ could be due to the higher intensity of ETT. The $\mathrm{HR}$ is a significant indicator of myocardial oxygen demand and autonomic regulation and balance. During exercise, there is a cardiovascular adaptation to accommodate the working muscles (metabolic demand) and skin blood flow (thermoregulatory demands) and maintain BP through the organs, so the HR is increased due to the suppression of the parasympathetic tone and the stimulation of the sympathetic system [23]. In Sousa et al.'s study [22] also, the correlation between maximum $\mathrm{HR}(r=0.68)$ and between maximum BP $(r=0.48)$ was assessed. In alignment with our results, Kristjánsdóttir et al.'s study [12] showed a moderate correlation between these variables during $6 \mathrm{MWT}$ and symptom-limited-graded exercise $(r=0.64)$ for maximum HR and $(r=0.52)$ for maximum systolic BP. Maximum HR during the $6 \mathrm{MWT}$ in the present study corresponded to $86 \%$ of that achieved during ETT, which represents a moderate-to-high intensity exercise that was confirmed in previous studies in a group of healthy middle-aged and older adults $[6,24]$. As most patients admitted to CR centers are elderly, these same results indicate that 6MWT can be a well-tolerated tool correlated with ETT that patients can perform without the fear of instability or falling off the treadmill. Gremeaux et al.'s study [25] reported that maximum HR during the $6 \mathrm{MWT}$ corresponded to $78 \%$ of that achieved during CPET in patients with stable coronary artery disease referred to the CR department. In CR programs, the intensity of the exercise is commonly determined using the target HR, estimating from CPET or ETT, however; the physiologic of daily activity is different and more compatible with 6MWT as a 
functional waking test. 6MWT also has the advantage of being more accessible and easily performed, and therefore can be used several times during the sessions for decision-making about ongoing treatment process and program intensity. This simple functional test exploring different effort intensities could be used as a complement to the ETT when starting a cardiac rehabilitation program and then as an alternative to repeated ETT to update optimal exercise intensity regularly during rehabilitation programs. 6MWT is not a substitute for the ETT, but a useful alternative instrument for assessing exercise capacity. May have benefits in providing information for individualized exercise prescription for those patients who enter a CR program without an entry graded exercise test and also as a functional and inexpensive outcome measures for several reassessments during the treatment to have a document-based decision-making about the need for continuing the program or discharging the patient.

The main limitation of this study is that MET attained during ETT as a reference test used to prove 6MWT validity is an estimation, and therefore probably not equal to directly measured MET during CPET. However, this was due to more accessibility of CR centers to ETT than CPET. The findings of this study are helpful for healthcare professionals and for the patients, as the test is easy to administer and also well tolerated by patients. However, there is a need for further research investigating 6MWT validity through the time and after passing CR as an outcome measure, means responsiveness.

\section{CONCLUSIONS}

The results from this study suggest that 6MWT is a valid and effective tool for assessing functional capacity for prescribing exercise in a group of postoperative CABG participants admitted to CR units.

\section{ACKNOWLEDGEMENTS}

The authors would like to thank the staff of the cardiac rehabilitation centers for their support during assessments and all the participants for contributing to this study.

Funding. This study was supported by grants from the Musculoskeletal Rehabilitation Research Center, Ahvaz Jundishapur University of Medical Sciences, Ahvaz, Iran (grant number: PHT-9923). No Rapid Service Fee was received by the journal for the publication of this article.

Authorship. All named authors meet the International Committee of Medical Journal Editors (ICMJE) criteria for authorship for this article, take responsibility for the integrity of the work as a whole, and have given their approval for this version to be published.

Disclosures. Maryam A. Saba, Shahin Goharpey, Behrouz Attarbashi Moghadam, Reza Salehi, and Mostafa Nejatian declare that they have no conflicts of interest.

Compliance with Ethics Guidelines. The Medical Ethics Committee of the Ahvaz Jundishapur University of Medical Sciences have approved the study protocols (IR. AJUMS.REC.1399.582). Written informed consent was obtained from all study subjects. The study was performed in accordance with the Declaration of Helsinki 1964 and its later amendments.

Data Availability. The datasets generated and/or analyzed during the current study are available from the corresponding author on reasonable request.

Open Access. This article is licensed under a Creative Commons Attribution-NonCommercial 4.0 International License, which permits any non-commercial use, sharing, adaptation, distribution and reproduction in any medium or format, as long as you give appropriate credit to the original author(s) and the source, provide a link to the Creative Commons licence, and indicate if changes were made. The images or other third party material in this article are included in the article's Creative Commons licence, unless indicated otherwise in a credit line to the material. If material is not included 
in the article's Creative Commons licence and your intended use is not permitted by statutory regulation or exceeds the permitted use, you will need to obtain permission directly from the copyright holder. To view a copy of this licence, visit http://creativecommons.org/licenses/by$\mathrm{nc} / 4.0 /$.

\section{REFERENCES}

1. Verrill DE, Barton C, Beasley W, Lippard M, King CN. Six-minute walk performance and quality of life comparisons in North Carolina cardiac rehabilitation programs. Heart Lung J Crit Care. 2003;32(1):41-51.

2. The minimum standard for those attending a Center-based program is: Measure of exercise capacity (e.g., cardiopulmonary exercise test (CPET), 6-minute walk test (6MWT), incremental shuttle walk test (ISWT). Measure of quality of life. http://www.heartonline.org.au/articles/exercise/ exercise-assessment\#outcome-measures.

3. ATS Committee on Proficiency Standards for Clinical Pulmonary Function Laboratories. ATS statement: guidelines for the six-minute walk test. Am J Respir Crit Care Med. 2002;166(1):111-7. https:// doi.org/10.1164/ajrccm.166.1.at1102. Erratum in: Am J Respir Crit Care Med. 2016;193(10):1185.

4. American Thoracic Society; American College of Chest Physicians. ATS/ACCP Statement on cardiopulmonary exercise testing. Am J Respir Crit Care Med. 2003;167(2):211-77. https://doi.org/10. 1164/rccm.167.2.211. Erratum in: Am J Respir Crit Care Med. 2003 May 15;1451-2.

5. Fletcher GF, Balady GJ, Amsterdam EA, Chaitman B, Eckel R, Fleg J, et al. Exercise standards for testing and training: a statement for healthcare professionals from the American Heart Association. Circulation. 2001;104(14):1694-740.

6. Sperandio EF, Arantes RL, Matheus AC, Silva RP, Lauria VT, Romiti M, et al. Intensity and physiological responses to the 6-minute walk test in middle-aged and older adults: a comparison with cardiopulmonary exercise testing. Braz J Med Biol Res. 2015;48(4):349-53.

7. Hill J, Timmis A. Exercise tolerance testing. BMJ Clin Res Ed. 2002;324(7345):1084-7.

8. Sharma K, Kohli P, Gulati M. An update on exercise stress testing. Curr Probl Cardiol. 2012;37(5): 177-202.
9. Bruce RA, Kusumi F, Hosmer D. Maximal oxygen intake and nomographic assessment of functional aerobic impairment in cardiovascular disease. Am Heart J. 1973;85(4):546-62.

10. Fletcher GF, Ades PA, Kligfield P, Arena R, Balady GJ, Bittner VA, et al. Exercise standards for testing and training: a scientific statement from the American Heart Association. Circulation. 2013;128(8):873-934.

11. Solway S, Brooks D, Lacasse Y, Thomas S. A qualitative systematic overview of the measurement properties of functional walk tests used in the cardiorespiratory domain. Chest. 2001;119(1):256-70.

12. Kristjansdottir A, Ragnarsdottir M, Einarsson MB, Torfason B. A comparison of the 6-minute walk test and symptom limited graded exercise test for phase II cardiac rehabilitation of older adults. J Geriatr Phys Ther. 2004;27(2):65.

13. Ngai SP, Jones AY, Jenkins SC. Regression equations to predict 6-minute walk distance in Chinese adults aged 55-85 years. Hong Kong Physiother J. 2014;32(2):58-64.

14. Fiorina C, Vizzardi E, Lorusso R, Maggio M, De Cicco G, Nodari S, et al. The 6-min walking test early after cardiac surgery. Reference values and the effects of rehabilitation programme. Eur J Cardiothorac Surg. 2007;32(5):724-9.

15. Bellet RN, Adams L, Morris NR. The 6-minute walk test in outpatient cardiac rehabilitation: validity, reliability and responsiveness-a systematic review. Physiotherapy. 2012;98(4):277-86.

16. Bittner V. Role of the 6-minute walk test in cardiac rehabilitation. Cardiac rehabilitation. Berlin: Springer; 2007. p. 131-9.

17. Ferguson B. ACSM's guidelines for exercise testing and prescription 9th Ed. 2014. J Can Chiropr Assoc. 2014;58(3):328.

18. Suaya JA, Shepard DS, Normand SL, Ades PA, Prottas J, Stason WB. Use of cardiac rehabilitation by Medicare beneficiaries after myocardial infarction or coronary bypass surgery. Circulation. 2007; 116(15):1653-62.

19. Kwok BC, Pua YH, Mamun K, Wong WP. The minimal clinically important difference of sixminute walk in Asian older adults. BMC Geriatr. 2013;13:23.

20. Hill K, Wickerson LM, Woon LJ, Abady AH, Overend TJ, Goldstein RS, et al. The 6-min walk test: responses in healthy Canadians aged 45 to 85 years. Appl Physiol Nutr Metab Physiologie appliquee, nutrition et metabolisme. 2011;36(5):643-9. 
21. Guazzi M, Dickstein K, Vicenzi M, Arena R. Sixminute walk test and cardiopulmonary exercise testing in patients with chronic heart failure: a comparative analysis on clinical and prognostic insights. Circ Heart Fail. 2009;2(6):549-55.

22. Pereira de Sousa LA, Britto RR, Ribeiro AL, Baracho SM, da Costa Val Barros V, Carvalho VT, et al. Sixminute walk test in patients with permanent cardiac pacemakers. J Cardiopulm Rehabil Prev. 2008;28(4):253-7.

23. Michael S, Graham KS, Davis GM. Cardiac autonomic responses during exercise and post-exercise recovery using heart rate variability and systolic time intervals-a review. Front Physiol. 2017;8:301.

24. Kervio G, Carre F, Ville NS. Reliability and intensity of the six-minute walk test in healthy elderly subjects. Med Sci Sports Exerc. 2003;35(1):169-74.

25. Gremeaux V, Deley G, Duclay J, Antoine D, Hannequin A, Casillas JM. The 200-m fast-walk test compared with the 6-min walk test and the maximal cardiopulmonary test: a pilot study. Am J Phys Med Rehabil. 2009;88(7):571-8. 